\title{
Intracranial gossypiboma 9 years after intracranial pressure bolt insertion: illustrative case
}

\author{
Ryan T. S. Loh, MBBChir, ${ }^{1}$ Tomasz Matys, MD, PhD, FRCR, ${ }^{2}$ Kieren S. J. Allinson, MBChB, FRCPath, EFN, ${ }^{3}$ and \\ Thomas Santarius, MD, PhD, FRCS(SN) ${ }^{4}$ \\ ${ }^{1}$ University of Cambridge School of Clinical Medicine, Cambridge, United Kingdom; and Departments of ${ }^{2}$ Radiology ${ }^{3}$ Pathology, and ${ }^{4}$ Neurosurgery, Cambridge University \\ Hospitals NHS Foundation Trust, Addenbrooke's Hospital, Cambridge, United Kingdom
}

\begin{abstract}
BACKGROUND Resorbable hemostatic agents left behind postoperatively occasionally result in granulomatous space-occupying lesions known as "gossypibomas." The authors report a case of an intracranial gossypiboma, which is exceedingly rare and frequently radiologically indistinguishable from other lesions.

OBSERVATIONS A 35-year-old woman presented with a generalized tonic-clonic seizure and subsequent left-sided hemiparesis. Magnetic resonance imaging showed an enhancing lobulated lesion subjacent to a right frontal burr hole, surrounded by vasogenic edema with mass effect and midline shift. Nine years earlier, she had had a triple bolt inserted to monitor intracranial pressure after sustaining a traumatic brain injury. Surgicel was used to control bleeding during insertion. Colocation of the lesion with the position of triple bolt 9 years earlier raised suspicion for gossypiboma. However, the minor nature of the surgery and the length of time since surgery to presentation placed this case well outside the range of cases reported in the literature. The lesion was resected en bloc with no recurrence 18 months later. Histological examination revealed the presence of foreign material. However, given its minute size, confirming its nature was not possible.
\end{abstract}

LESSONS The authors show that gossypibomas can occur following a relatively minor procedure and remain clinically and radiologically silent for much longer than previously reported.

https://thejns.org/doi/abs/10.3171/CASE21479

KEYWORDS gossypiboma; foreign body granuloma; textiloma; Surgicel; ICP bolt

Foreign body granulomas, also known as "gossypibomas," are known to occur after retention of nonabsorbable as well as absorbable material used during surgery. ${ }^{1,2}$ They usually present radiologically as peripherally enhancing lesions that are frequently indistinguishable from other much more frequently occurring masses, such as tumors and abscesses, making a definitive diagnosis before histopathological investigation difficult. $^{3}$

We report a case of a patient with gossypiboma presenting 9 years after intracranial pressure (ICP) monitoring bolt insertion. This remarkably long lag phase combined with the minor nature of the initial procedure makes this case particularly unusual.

\section{Illustrative Case}

A 35-year-old woman sustained a severe traumatic brain injury in a road traffic accident. During hospitalization, she underwent insertion of a triple-bolt monitoring device consisting of a Licox brain tissue oxygenation probe (Integra LifeSciences), microdialysis catheter, and Codman intraparenchymal ICP probe (Integra LifeSciences), ${ }^{4}$ which is performed routinely in our unit. Small superficial bleeding from the dura and brain surface during insertion was controlled with Surgicel (Ethicon). She did not undergo any other surgical interventions and subsequently made a full recovery.

Nine years later, she presented with loss of consciousness and generalized tonic-clonic seizure, and subsequently she developed left-sided hemiparesis. She underwent computed tomography and subsequently magnetic resonance imaging (MRI), which revealed an avidly enhancing lobulated lesion with T2-hypointense center subjacent to the old right frontal burr hole, surrounded by a large area of vasogenic edema with mass effect and midline shift 

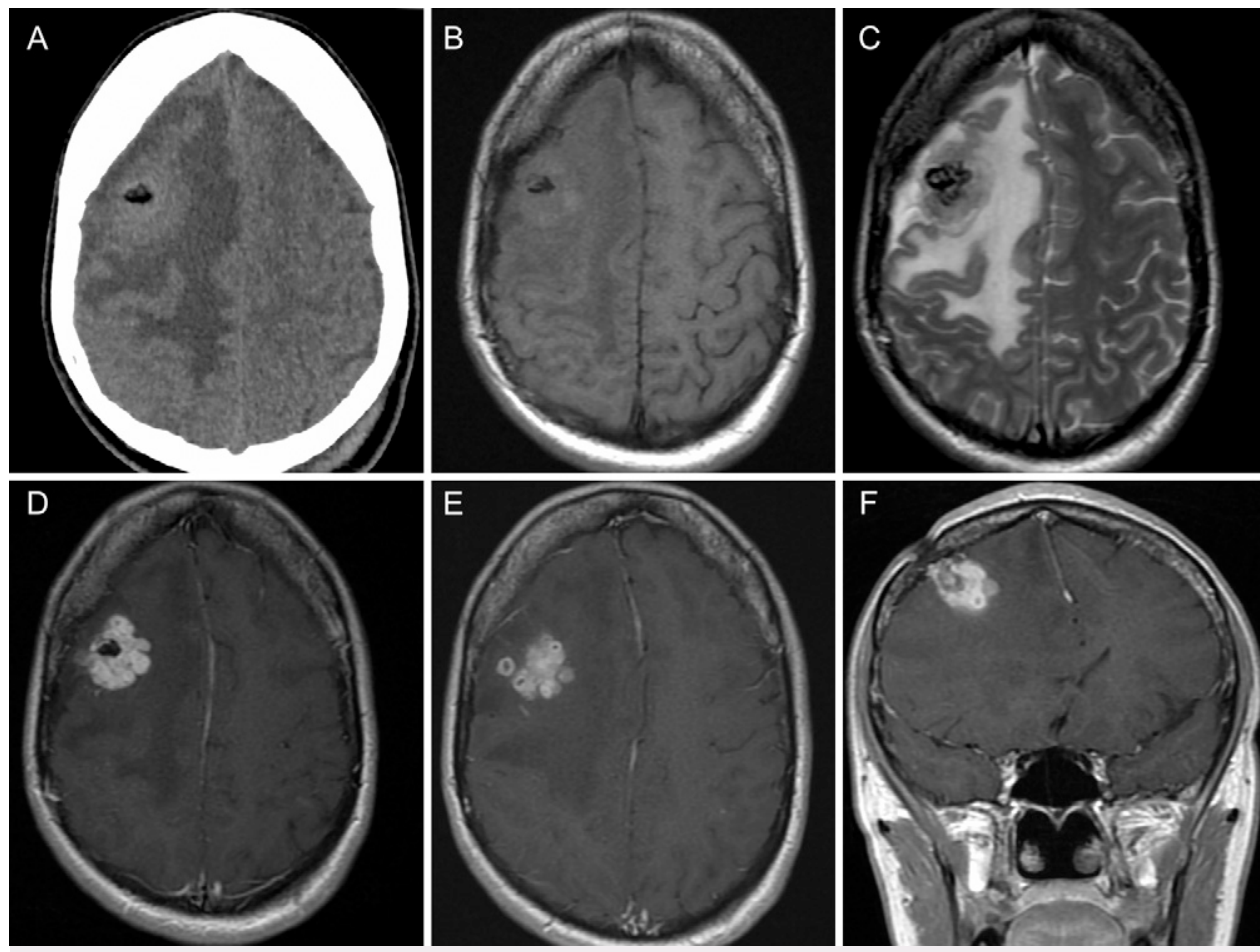

FIG. 1. Imaging showed a lesion in the right frontal lobe surrounded by extensive vasogenic edema with moderate mass effect. On computed tomography $(\mathrm{CT})(\mathbf{A})$, the lesion showed a hypointense area in the center with average density values of approximately -50 Hounsfield units, suggestive of the presence of fat. However, on MRI, the lesion had signal characteristics discordant from the CT findings, with a focus of low T1 signal centrally (B) and a corresponding larger area of low T2 signal (C); high T1 and T2 signals would be expected for fat. After administration of gadolinium contrast medium, the lesion showed avid enhancement throughout sparing the central T1-hypointense focus (D). The enhancement pattern was unusual with lobulated multilocular appearance at the periphery of the lesion $(\mathbf{E})$ and extended to the dura subjacent to the old frontal burr hole (F).

(Fig. 1). The patient was commenced on dexamethasone $4 \mathrm{mg}$ twice daily. Her seizures stopped, and the left-sided weakness improved significantly, reverting almost to normal. Her only other medical history of note was heterozygotic Fabry disease carrier status (N215S mutation) discovered as part of family screening, with no clinical manifestations.

A more detailed history revealed that 2 years earlier, she had two seizure-like episodes associated with strenuous exercise, which were diagnosed at the time as vasovagal attacks.

Colocation of the lesion with the position of the triple bolt inserted 9 years earlier raised a suspicion of a gossypiboma. However, the relatively minor and uncomplicated nature of the surgery, in particular the length of time elapsed since the procedure, was unusual, raising a possibility of a neoplasm. The patient was discussed at a multidisciplinary team meeting, and surgical resection was deemed the most appropriate management option. During surgery, the scar of the burr hole was found to be connected via the dura with the lesion, which was firm, and fibrous in places with gliotic reaction in the surrounding brain (Fig. 2). The lesion was removed en bloc. Postoperative MRI showed complete resection of the lesions, and 15-month follow-up MRI revealed no recurrence.

Histopathological examination (Fig. 3) revealed features of an acute-on-chronic inflammatory (predominantly extraaxial) lesion with the presence of a minuscule amount of foreign material consistent with Surgicel, confirming the suspicion of gossypiboma.

\section{Discussion \\ Observations}

Our case is highly unusual because most reported cases involve previous craniotomy, with no reports thus far of gossypibomas forming after minor procedures, such as insertion of an ICP monitoring device via a burr hole. Another noteworthy feature is its pattern of progression. The time frame for clinical presentations of retained foreign bodies vary greatly, ranging from days to decades postoperatively, though a majority present days to months after operation. ${ }^{3,5-7}$ Our patient underwent follow-up MRI 2 and 3 years after her traumatic brain injury, which revealed a small amount of T2hypointense material along the trajectory of the ICP probe, interpreted as sequelae of a small hemorrhage associated with probe insertion, but no unexpected findings. Therefore, a reasonable deduction would be that the lesion started to develop at least 3 years postoperatively, ultimately resulting in major symptoms 9 years postoperatively. Such a long lag phase between the initial procedure and presentation is extremely rare. ${ }^{8-12}$ The reasons for activation of the inflammatory process following such long lag periods are unknown. 

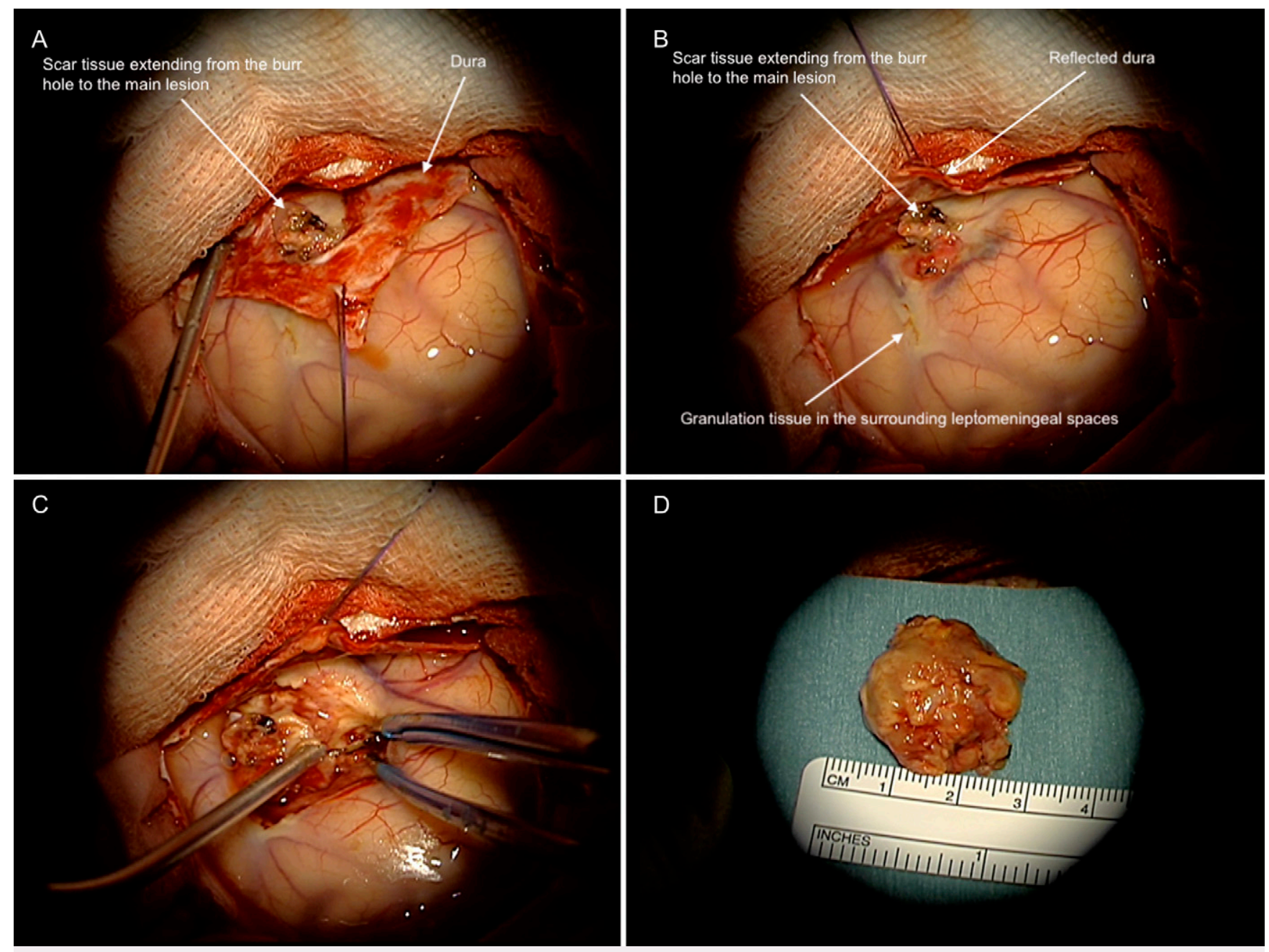

FIG. 2. Intraoperative images showing removal of the lesion en bloc. A bone flap containing the old burr hole is removed, and burr hole scar tissue is shown crossing the dura $(\mathbf{A})$. The dura is first reflected, exposing the surface of the lesion (B). The granuloma is then dissected from the surrounding gliotic brain (C). The removed granuloma is approximately $2.5 \mathrm{~cm}$ in diameter (D).

The minute foreign body contained in the specimen did not allow its unequivocal identification. The potential candidates include fragments of the plastic related to the ICP bolt and other nonmetallic fragments as well as Surgicel. Surgicel-elicited granulomas have been reported across many systems, but those occurring in the brain are especially rare, especially given how ubiquitously hydrocellulose products are used in cranial neurosurgery. ${ }^{13}$ Previously described intracranial cases histologically show signs of necrosis and a mixed picture of acute and chronic inflammation, in keeping with our findings. ${ }^{1}$ As in our case, gossypibomas are difficult to distinguish from neoplasms, which may pose a particular problem in the setting of suspected tumor recurrence after a prior resection. ${ }^{6}$ Interestingly, our samples do show focal foreign body-type multinucleated giant cells, suggesting that the granuloma grew from the dural surface into the brain or at least that a connection with the meninges was maintained along with its extraaxial blood supply, thus at least partially remaining outside the blood-brain barrier. MRI appearances showing extension of the lesion to the dural surface (Fig. 1F) also support this hypothesis.
Whether the gossypiboma is related to the patient's Fabry disease is unknown. Fabry disease is not known to result in granulomas, but, being a lysosomal storage disorder, the accumulation of Gb3 has been reported to promote inflammation, cytokine secretion, and apoptosis. ${ }^{14}$ However, because there is still much unknown about the role of classic inflammatory pathways in the pathogenesis of Fabry disease and its clinical relevance, logging this case in the literature may help other clinicians investigate this further in case they too come across a granuloma in a patient with Fabry disease.

\section{Lessons}

Intracranial gossypibomas are remarkably rare and in most previous reports developed within months after a craniotomy. Our case is highly unusual, given its association with a minor burr hole procedure of intracranial bolt insertion and the very long lag period. When a lesion appears at the site where a previous procedure was performed, gossypibomas should be considered in the differential diagnosis. 


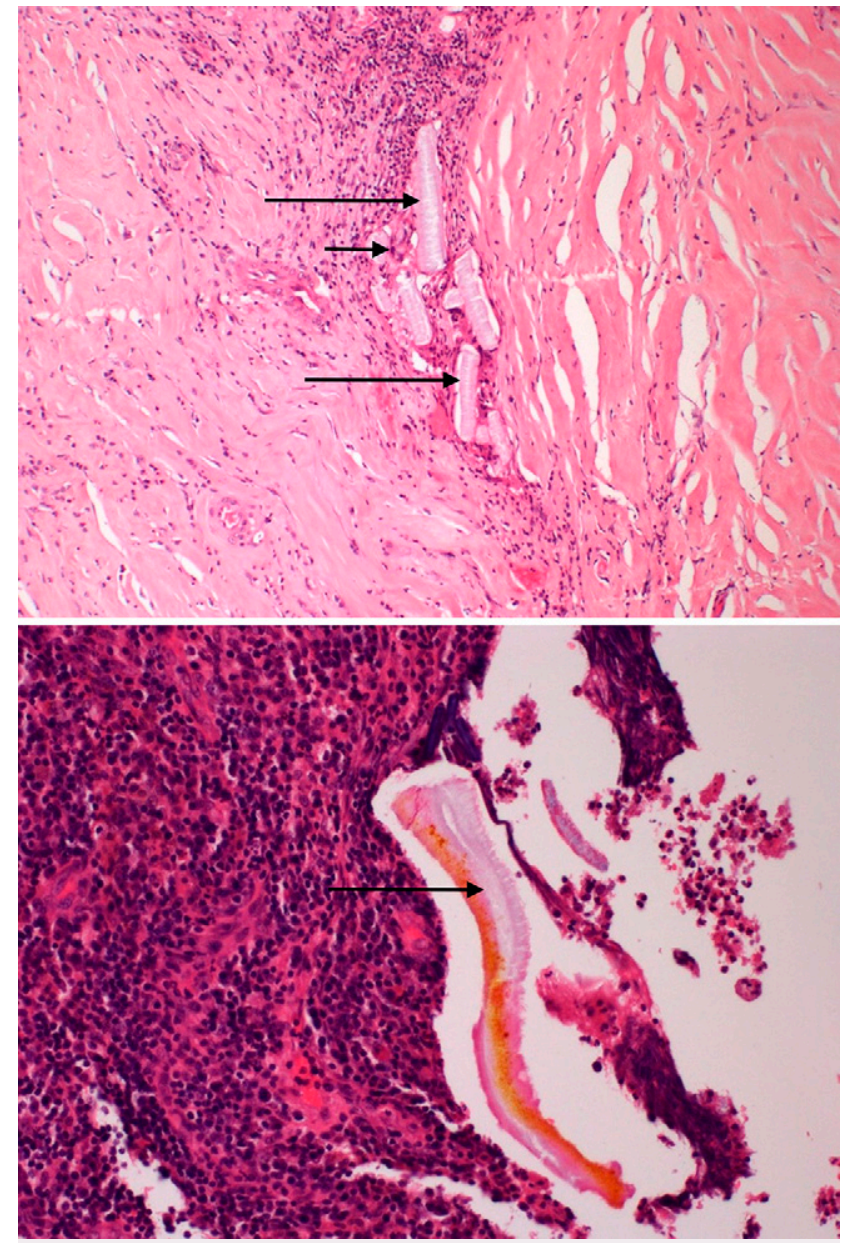

FIG. 3. Histopathological samples of lesion showing fragments of foreign material equivocally consistent with Surgicel (long arrows), with foreign body giant cell response (short arrow) and surrounding fibrosis (upper; hematoxylin and eosin [H\&E] stain; original magnification, $\times 10$ ). Also shown is evidence of chronic inflammation with associated chronic, lymphoplasmacytic cell infiltrate (lower; H\&E stain; original magnification, $\times 20)$.

\section{References}

1. Ribalta T, McCutcheon IE, Neto AG, et al. Textiloma (gossypiboma) mimicking recurrent intracranial tumor. Arch Pathol Lab Med. 2004;128(7):749-758.

2. Kim AK, Lee EB, Bagley LJ, Loevner LA. Retained surgical sponges after craniotomies: imaging appearances and complications. AJNR Am J Neuroradiol. 2009;30(6):1270-1272.

3. Bilginer B, Yavuz K, Agayev K, Akbay A, Ziyal IM. Existence of cotton granuloma after removal of a parasagittal meningioma: clinical and radiological evaluation: a case report. Kobe $J$ Med Sci. 2007;53(1-2):43-47.
4. Hutchinson PJA, Hutchinson DBA, Barr RHH, Burgess F, Kirkpatrick PJ, Pickard JD. A new cranial access device for cerebral monitoring. Br J Neurosurg. 2000;14(1):46-48.

5. Martins MCB, Amaral RPG, Andrade CS, Lucato LT, Leite $C$ da C. Magnetic resonance imaging findings of intracranial gossypiboma: a case report and literature review. Radiol Bras. 2009;42(6):407-409.

6. Lin B, Yang H, Cui M, Li Y, Yu J. Surgicel application in intracrania hemorrhage surgery contributed to giant-cell granuloma in a patient with hypertension: case report and review of the literature. World $J$ Surg Oncol. 2014;12:101.

7. Akpinar A, Ucler N, Ozdemir CO. Textiloma (gossypiboma) mimicking recurrent intracranial abscess. BMC Res Notes. 2015;8:390.

8. Dzenitis AJ, Kalsbeck JE. Chronic brain abscess discovered 31 years after intracerebral injury by missile: report of a case. J Neurosurg. 1965;22:169-171.

9. Jang SW, Kim SJ, Kim SM, et al. MR spectroscopy and perfusion MR imaging findings of intracranial foreign body granuloma: a case report. Korean J Radiol. 2010;11(3):359-363.

10. Feldman RP, Marcovici A, Suarez M, Goodrich JT. Foreign body granuloma mimicking intracranial meningioma: case report and review of the literature. Neurosurgery. 1999;44(4):855-858.

11. Hasturk $A E$, Basmaci $M$. Foreign body granuloma mimicking recurrent intracranial tumor: a very rare clinical entity. Acta Med Iran. 2013;51(11):816-818.

12. Peloquin P, Vannemreddy PSSV, Watkins LM, Byrne RW. Intracranial cotton ball gossypiboma mimicking recurrent meningioma: report of a case with literature review for intentional and unintentional foreign body granulomas. Clin Neurol Neurosurg. 2012;114(7):1039-1041.

13. Buckley SC, Broome JC. A foreign body reaction to Surgicel mimicking an abscess or tumour recurrence. $\mathrm{Br} \mathrm{J}$ Neurosurg. 1995;9(4):561-563.

14. Rozenfeld P, Feriozzi S. Contribution of inflammatory pathways to Fabry disease pathogenesis. Mol Genet Metab. 2017;122(3):19-27.

\section{Disclosures}

The authors report no conflict of interest concerning the materials or methods used in this study or the findings specified in this paper.

\section{Author Contributions}

Conception and design: Loh, Santarius. Acquisition of data: all authors. Analysis and interpretation of data: Loh, Matys, Allinson, Santarius. Drafting the article: Loh, Matys, Allinson, Santarius. Critically revising the article: all authors. Reviewed submitted version of manuscript: Loh Santarius. Approved the final version of the manuscript on behalf of all authors: Loh. Administrative/technical/material support: Loh, Santarius. Study supervision: Santarius.

\section{Supplemental Information}

Previous Presentations

This case report was subject to full peer review and was accepted for poster presentation at the ASiT Annual Conference 2021: Excelling in Adversity international conference, March 5-7, 2021.

\section{Correspondence}

Ryan T. S. Loh: Imperial College Healthcare NHS Trust, Cambridge, United Kingdom. tyngshen@yahoo.com; ryan.loh@nhs.net. 